Investigación Descriptiva, Correlacional o Cualitativa

Pensar en Movimiento:

Revista de Ciencias del Ejercicio y la Salud

ISSN 1659-4436

Vol. 14, No.1, pp. 1- 14

Cierre al 30 de junio, 2016

\title{
ACTIVIDAD FÍSICA Y OTROS HÁBITOS DE VIDA DE ESTUDIANTES DE MEDICINA DE LA UNIVERSIDAD DE COSTA RICA
}

\section{PHYSICAL ACTIVITY AND OTHER LIFESTYLE HABITS OF MEDICAL STUDENTS AT THE UNIVERSITY OF COSTA RICA}

\author{
Sergio Jiménez Morgan, Lic. 1(A,B,C,D,E); Jesennia Hernández Elizondo, Ph.D. 2(A,D,E) \\ sergio.jimenezmorgan@ucr.ac.cr; jesennia.hernandez@ucr.ac.cr \\ ${ }^{1}$ Escuela de Medicina, Universidad de Costa Rica, San José, Costa Rica \\ ${ }^{2}$ Escuela de Educación Física y Deportes, Universidad de Costa Rica, San José, Costa Rica
}

Manuscrito recibido: 24/05/2015; reenviado: 19/08/2015, 4/11/2015; aceptado: 26/11/2015; publicado: $01 / 02 / 2016$

Doi: http://dx.doi.org/10.15517/pensarmov.v\%vi\%i.19294

\section{RESUMEN}

Jiménez-Morgan, S., y Hernández-Elizondo, J. (2016). Actividad física y otros hábitos de vida de estudiantes de Medicina de la Universidad de Costa Rica. PENSAR EN Movimiento: Revista de Ciencias del Ejercicio y la Salud, 14(1), 1-14. Las médicas y los médicos desempeñan un rol como modelos sociales, y sus hábitos personales influyen en su ejercicio profesional y la calidad de la prevención primaria que realizan. El objetivo principal de este estudio fue determinar si, como médicas y médicos en formación, las y los estudiantes de medicina de la Universidad de Costa Rica cumplen con las recomendaciones nacionales e internacionales en componentes claves de un estilo de vida saludable. Se aplicaron cuestionarios anónimos, durante el segundo semestre del año 2014, a estudiantes de primero a quinto año de carrera ( $n$ = 206). Por medio de dichos documentos fueron recolectados algunos datos personales, así como información acerca de hábitos de tabaquismo, ingesta de alcohol, frecuencia y cantidad de consumo de frutas y vegetales, y actividades físicas practicadas. Se determinó que la mayoría de los participantes ingería bebidas alcohólicas (52,91\%), con una baja prevalencia de tabaquistas activos (3,39\%). Además, si bien cumplieron las recomendaciones nacionales e internacionales para el consumo de frutas y vegetales, el $40 \%$ de los participantes no realizaba ningún ejercicio físico, y entre quienes sí lo hacían diariamente, el 68,93\% dedicaba en promedio solamente 7,78 minutos a dicha actividad. Finalmente, quienes se ejercitaban una vez a la semana dedicaban más tiempo al estudio y menos tiempo a ver televisión. En conclusión, 
urge implementar estrategias de promoción del ejercicio en esta población universitaria, para prevenir que estos futuros profesionales desarrollen sobrepeso, obesidad y enfermedades crónicas asociadas a la baja actividad física.

Palabras claves: actividad física; ejercicio; nutrición; estudiantes medicina; Costa Rica

\begin{abstract}
Those engaged in medical sciences are social models. For this reason, their daily habits influence their professional practice and the primary prevention they carry out. The main purpose of this research was to determine whether this group of medicine training students, all from University of Costa Rica, met national and international recommendations regarding health and wellness regulations. In 2014, second semester, anonymous questionnaires were applied to all undergraduate medical students from first year to seniors $(n=216$.) Based on this information, some personal data was gathered such as smoking habits, alcohol intake, fruit and vegetable consumption, and the amount of physical activities they practiced. According to this evidence, $52.91 \%$ drank alcohol beverages while the prevalence of active smokers was low (3.39\%). Furthermore, although they accomplished the national and international standards related to fruit and vegetable consumption, $40 \%$ of the subjects did not exercise in any way. Among those who did exercise daily, $68.9 \%$ invested only 7.8 minutes per day. Among other findings, those who performed some physical activity at least once a week devoted more time to studying than to watching television. In brief, fitness and health promotion programs should target these future professionals to prevent risk factors such as overweight, obesity, and chronic diseases associated with a sedentary lifestyle.

English translation by Jeannette Soto Segura
\end{abstract}

Keywords: physical activity; exercise; nutrition; medical students; Costa Rica

Entre 1980 y 2013 la cifra mundial de personas con sobrepeso y obesidad incrementó de 857 millones a 2,1 billones, lo cual representó un aumento en la prevalencia de $27,5 \%$ en adultos y 47,1 \% en niños ( $\mathrm{Ng}$ et al., 2014). De acuerdo con ese mismo estudio, en el año 2013 la proporción de obesidad en adultas y adultos costarricenses de más de 20 años de edad fue de $15,4 \%$ en hombres y $28,8 \%$ en mujeres, mientras que para sobrepeso y obesidad combinados las cifras ascendieron a 55,2 \% y 66,5 \%, respectivamente. Estos valores concuerdan con lo reportado por la World Health Organization (WHO) en sus más recientes Estadísticas Sanitarias Mundiales, que señalan una prevalencia de obesidad en Costa Rica del $20,9 \%$ y $28,3 \%$ para hombres y mujeres mayores a 20 años de edad, respectivamente $(\underline{\mathrm{WO}}$, 2014).

Tanto el sobrepeso como la obesidad han sido asociados a un mayor riesgo de desarrollar enfermedades crónicas, por ejemplo: hipertensión arterial, dislipidemias, diabetes mellitus tipo 2, enfermedad arterial coronaria, cardiopatías, apnea del sueño y algunos tipos de cáncer (Jensen et al., 2014). En contraste, un mayor consumo de frutas y vegetales está asociado con un menor riesgo de desarrollar enfermedades no transmisibles y prevenibles como el sobrepeso y la obesidad, además disminuye el riesgo de muerte por enfermedades crónicas como las ya citadas (Oyebode, Gordon-Dseagu, Walker \& Mindell, 2014). Asimismo, la 
evidencia actual muestra que las personas físicamente activas presentan mejor perfil biológico frente a enfermedades cardiovasculares y diabetes tipo 2, así como mayor capacidad cardiorrespiratoria, muscular y salud ósea en comparación con quienes no realizan actividad física (Garber, Blissmer, Deschenes, Franklin, Lamonte, Lee, Nieman, Swain, 2011). La Organización Panamericana de la Salud (OPS), citando a la WHO, considera que si cuatro factores de riesgo conductuales fueran eliminados (lo cual es posible, ya que son comportamientos modificables) como son el consumo nocivo de alcohol, el sedentarismo, el tabaquismo y los hábitos inadecuados de alimentación, "se podría prevenir el $80 \%$ de las cardiopatías, los accidentes cerebrovasculares y los casos de diabetes de tipo 2 y más del $40 \%$ de los cánceres" (OPS, 2011, p. 4).

Las y los médicos desempeñan un papel preponderante en la prevención primaria del sobrepeso, la obesidad y los hábitos inadecuados de alimentación, por lo tanto fungen como modelos sociales y sus propias prácticas impactan negativa o positivamente su credibilidad ante los pacientes (Lobelo \& de Quevedo, 2014; Fie, Norman \& While, 2012). El tabaquismo en médicos y la relación de este hábito con la práctica profesional también ha sido objeto de estudio en años recientes (Duaso, McDermott, Mujika, Purssell \& While, 2014). Asimismo, las y los estudiantes de medicina de la Universidad de Costa Rica, como profesionales en formación, cumplen ese rol social ante sus pacientes durante sus prácticas hospitalarias, así como en sus familias y otros círculos sociales. Más aún, con base en la evidencia que existe actualmente acerca de los beneficios para la salud física y mental del ejercicio y la actividad física, las y los médicos se encuentran en la obligación ética de prescribir ejercicio a sus pacientes (Chakravarthy, Joyner \& Booth, 2002).

Por dichos motivos, es importante conocer cuáles son los hábitos de alimentación, tabaquismo, ingesta de alcohol y ejercicio físico de esta población, ya que se ha demostrado que existe una correlación positiva entre un estilo de vida saludable y una mejor actitud hacia la promoción de la salud y la prevención primaria, tanto en su etapa de estudiantes como en el ejercicio de sus profesiones (Duperly et al., 2009).

\section{METODOLOGÍA}

Sujetos. Estudio transversal con estudiantes de primero a quinto año de la carrera "Bachillerato en Ciencias Médicas y Licenciatura en Medicina y Cirugía" de la Universidad de Costa Rica, durante el segundo curso lectivo 2014 (agosto a diciembre). El instrumento fue aplicado a estudiantes que durante ese periodo se encontraran matriculados en la Sede Rodrigo Facio (San Pedro de Montes de Oca, San José, Costa Rica) y en el Hospital Rafael Ángel Calderón Guardia (San José, Costa Rica). En total participaron 206 estudiantes, seleccionados por muestreo no probabilístico entre un total de 662 estudiantes de primero a quinto año de carrera inscritos oficialmente en ese periodo. La cantidad total de estudiantes que completó el instrumento por cada año de carrera se detalla en la tabla 1. 
Tabla 1

Distribución de la muestra por sexo y edad, según año de carrera

\begin{tabular}{ccccc}
\hline $\begin{array}{c}\text { Año de } \\
\text { carrera }\end{array}$ & $\begin{array}{c}\mathrm{N} \text { por } \\
\text { año }\end{array}$ & Mujeres & Hombres & Edad (en años) \\
\hline 1 & 38 & $21(55.26 \%)$ & $17(44.74 \%)$ & $18.63[0.71]$ \\
2 & 77 & $42(54.55 \%)$ & $35(45.45 \%)$ & $21.10[0.71]$ \\
3 & 27 & $14(51.85 \%$ & $13(48.15 \%)$ & $22.19[2.82]$ \\
4 & 35 & $17(48.57 \%)$ & $18(51.43 \%)$ & $23.66[1.41]$ \\
5 & 29 & $15(51.72 \%)$ & $14(48.28 \%)$ & $24.41[2.12]$ \\
\hline
\end{tabular}

Fuente: elaboración propia

Para poder participar, debían encontrarse activos en la carrera en el periodo de la realización del estudio. No se establecieron restricciones de género ni edad. Por otra parte, fueron excluidas las personas que pertenecieran a otra carrera, y quienes hubieran llenado el instrumento en forma incompleta.

Instrumentos. Cuestionario en formato impreso, modificado a partir de HernándezElizondo (2009), en el cual se incluyeron los siguientes apartados: datos personales como edad, sexo y año de carrera del sujeto; hábitos de tabaquismo e ingesta de alcohol; frecuencia de consumo de alimentos (FFQ), y cuestionario de actividad física cotidiana (PAQ).

Para el FFQ se escogieron 53 alimentos agrupados en vegetales, legumbres y frutas. La información solicitada consistió en el consumo o no de cada uno de los alimentos según el grupo, la frecuencia de consumo semanal y la cantidad de consumo en cada ocasión (expresada con medidas caseras). El FFQ empleado se basó en un cuestionario que fue utilizado y validado previamente por Hernández-Elizondo (2009), con valores de significación menores a 0.05 en las correlaciones de Spearman para diferentes grupos de alimentos.

EI PAQ empleado fue desarrollado a partir de cuestionarios que ya habían sido previamente utilizados y validados (Mariscal-Arcas et al., 2007; Hernández-Elizondo et al., 2009). Incluyó preguntas acerca de la realización de ejercicio físico, el tipo de ejercicio practicado, la frecuencia semanal y el tiempo dedicado (en minutos) en cada ocasión. Adicionalmente, los sujetos completaron un recuento de 24 horas de sus actividades cotidianas (en horas y minutos), para diferentes parámetros como la higiene personal, tareas domésticas, actividades pasivas (estudiar, usar la computadora, ver la televisión, escuchar música, entre otras), actividades dinámicas (videojuegos activos o exer-games, desplazamientos para ir de compras o por estudio) y otras actividades (tiempos de comida, actividades sociales, dormir, entre otras). Respecto a dicho instrumento, y para fines conceptuales en la presente investigación, "actividad física" fue considerada como cualquier movimiento corporal producido por la musculatura esquelética que resulte en gasto energético (Caspersen, Powell \& Christenson, 1985). Acorde con los mismos autores, "ejercicio" fue considerado como toda 
aquella actividad física planeada, estructurada y repetitiva, y cuyo objetivo es el mejoramiento o mantenimiento de la aptitud física (Caspersen et al., 1985).

Los instrumentos fueron aplicados durante los recesos de las lecciones teóricas de las y los estudiantes, con el fin de no interrumpir las actividades académicas ni hospitalarias. En forma verbal se les explicó, previamente, el objetivo principal de la investigación, así como el carácter voluntario y anónimo de su participación en el estudio. Se respetó cada caso que manifestara la negativa a colaborar.

En total se realizaron cuatro visitas a la Escuela de Medicina de la Sede Rodrigo Facio y cuatro al Hospital Rafael Ángel Calderón Guardia, con el fin de aplicar y completar la mayor cantidad posible de instrumentos.

Los cuestionarios fueron aplicados tanto a estudiantes hombres como mujeres, sin que se estableciera previamente una proporción entre ambos géneros, ya que los objetivos de la investigación no contemplaban buscar diferencias por sexo.

Análisis estadístico. Para las variables cuantitativas se calcularon medias, desviaciones estándar, distribuciones de normalidad, valores mínimos y máximos. En el análisis de variables cualitativas se utilizaron distribuciones de frecuencias y tests de $\mathrm{Chi}^{2}$. Para el análisis inferencial de la información obtenida con el FFQ se empleó la prueba $t$ de Student para una sola muestra. Finalmente, la información obtenida a partir del PAQ fue analizada mediante tests de $\mathrm{Chi}^{2}$ y análisis de varianza (ANOVA) de una vía para muestras independientes, así como pruebas post hoc (Tukey) cuando fue necesario.

Para todos los análisis se utilizó el paquete estadístico SPSS 20.0 (IBM Corp. Armonk, NY, USA). En cada test se estableció un nivel de significación de $p<0.05$.

\section{RESULTADOS}

No se encontraron diferencias significativas entre hombres y mujeres para las distintas variables medidas, por lo cual los resultados obedecieron al análisis del conjunto de los 206 sujetos que participaron en el estudio. En total, completaron satisfactoriamente los instrumentos 109 mujeres (52.91\%) y 97 hombres (47.09\%), con una edad media de $21.69 \pm 2.83$ años. La tabla 1 detalla la distribución por sexo y edades para cada año de carrera.

El $52.91 \%$ de los sujetos ingería bebidas alcohólicas, sin diferencias estadísticamente significativas entre los hombres y las mujeres que sí las consumían $\left(C^{2}{ }^{2}=1.709, p=0.191\right)$. La quinta parte de quienes lo hacían inició el consumo durante la etapa universitaria (21.10\%). Por el contrario, solo un 3.39 \% de la población afirmó que fumaba en ese momento, en su mayoría hombres $(n=6)$; solamente una mujer afirmó ser tabaquista activa al momento del estudio.

Los frijoles negros y rojos constituyeron las legumbres más consumidas $(46.67 \%$ y $29 \%$, respectivamente), mientras que el jugo de naranja correspondió a la fruta más ingerida (50.49 $\%)$. En relación con las verduras harinosas, las de mayor ingesta fueron el plátano maduro (44.83 \%) y la papa (43.77\%). Finalmente, la lechuga y el tomate fueron los vegetales más consumidos por los sujetos (13.91\% y $17.72 \%$, respectivamente).

La tabla 2 muestra las medias de consumo semanal de los sujetos para los diferentes grupos, en comparación con las recomendaciones planteadas en las Guías Alimentarias para 
Costa Rica (Ministerio de Salud de Costa Rica, 2011) y Dapcich, Salvador, Pérez, Aranceta y Serra (2004). Los sujetos consumieron cantidades significativamente mayores de frutas $(p=$ $0.001)$ y legumbres $(p=0.014)$, y cumplieron con los requerimientos mínimos de vegetales y verduras harinosas.

Tabla 2

Frecuencia de consumo de alimentos vs. recomendaciones semanales (veces/semana)

\begin{tabular}{ccccc}
\hline Alimento & $\begin{array}{c}\text { Frecuencia de } \\
\text { consumo } \\
\text { media semanal }\end{array}$ & $\begin{array}{c}\text { Recomendación } \\
\text { semanal a } \\
\text { (veces/semana) }\end{array}$ & $\mathrm{t}$ & Sig. \\
\hline Frutas & 26.20 & 21 & 3.482 & 0.001 \\
Vegetales & 14.81 & 14 & 1.048 & 0.296 \\
Legumbres & 8.74 & 7 & 2.468 & 0.014 \\
Verduras & 8.31 & 8 & 0.484 & 0.629 \\
harinosas & & & & \\
\hline
\end{tabular}

a Ministerio de Salud de Costa Rica (2011) y Dapcich, Salvador, Pérez, Aranceta, Serra (2004) Fuente: elaboración propia.

Cuatro de cada diez participantes del estudio no realizaban ningún ejercicio físico, y solamente una de cada cinco personas se ejercitaba a diario. Más del $65 \%$ de los sujetos caminaba como forma de ejercitarse, con una mayor proporción de quienes lo hacían a paso lento respecto a quienes lo hacían a paso rápido. Los deportes menos practicados fueron el yoga (3.88 \%), el baloncesto (3.39\%) y la natación (6.31\%). Por otra parte, un $87.86 \%$ de los estudiantes se desplazaba con mayor frecuencia en automóvil o autobús, contra un $10.68 \%$ que caminaba o manejaba bicicleta al trasladarse $\left(\mathrm{Chi}^{2}=161.340\right.$ con nivel de significancia $p<0.001$ ). Casi el $70 \%$ de los sujetos dedicaba en promedio únicamente 7.78 minutos diarios a realizar algún ejercicio, como muestra la tabla 3. 
Tabla 3

Tiempo diario (en minutos) dedicado al ejercicio físico

\begin{tabular}{cccccccc}
\hline Minutos & $\mathrm{N}(\%)$ & $\mathrm{MIN}$ & MAX & Media & $\mathrm{DS}$ & $\mathrm{F}$ & $\mathrm{P}$ \\
\hline 0 a 30 & $142(68.93 \%)$ & 0 & 30 & $7.78^{*}$ & 12.53 & 711.98 & $<0.001$ \\
31 a 60 & $33(16.02 \%)$ & 40 & 60 & $56.36 *$ & 7.42 & & \\
61 a 120 & $26(12.62 \%)$ & 90 & 120 & $105 *$ & 15.29 & & \\
121 a 180 & $5(2.43 \%)$ & 150 & 180 & 162 * & 16.43 & & \\
\hline
\end{tabular}

* Diferencias significativas $(p<0.05)$ entre todos los grupos, según el análisis post hoc (Tukey).

Finalmente, no existieron diferencias significativas en actividades diarias como higiene personal, tareas del hogar o actividades sociales, entre quienes no realizaban ejercicio físico y quienes sí lo hacían al menos una vez a la semana (tabla 4). Por el contrario, sí existieron diferencias significativas en cuanto al tiempo dedicado al estudio y a ver la televisión. Según se puede observar en las figuras 1 y 2, a partir del análisis estadístico post hoc (Tukey) se encontró que quienes realizaban ejercicio físico una vez a la semana invertían más tiempo en el estudio y veían menos la televisión.

Tabla 4

Tiempo destinado a actividades cotidianas, según frecuencia semanal de ejercicio físico

\begin{tabular}{ccccccc}
\hline & \multicolumn{7}{c}{ Frecuencia de ejercicio físico } & & \\
\cline { 2 - 5 } $\begin{array}{c}\text { Actividad } \\
\text { (min/día) }\end{array}$ & $\begin{array}{c}\text { No } \\
\text { realiza } \\
\text { AF }\end{array}$ & $\begin{array}{c}1 \\
\text { v/semana }\end{array}$ & $\begin{array}{c}2-3 \\
\text { v/semana }\end{array}$ & $\begin{array}{c}\text { A } \\
\text { diario }\end{array}$ & & P \\
\hline $\begin{array}{c}\text { Higiene } \\
\text { personal }\end{array}$ & 74.23 & 60.18 & 66.67 & 80.75 & 1.611 & 0.188 \\
Tareas del & 33.51 & 37.86 & 42.22 & 38.75 & 0.593 & 0.620 \\
hogar & & & & & & \\
Estudiar & 471.10 & 700.71 & 424.26 & 437.25 & 8.113 & $0.001^{*}$ \\
Ver TV & 39.52 & 13.57 & 42.80 & 53.63 & 4.120 & $0.007^{*}$ \\
Actividades & 108.43 & 68.21 & 93.98 & 106.75 & 1.193 & 0.313 \\
sociales & & & & & & \\
\hline
\end{tabular}

Fuente: elaboración propia 


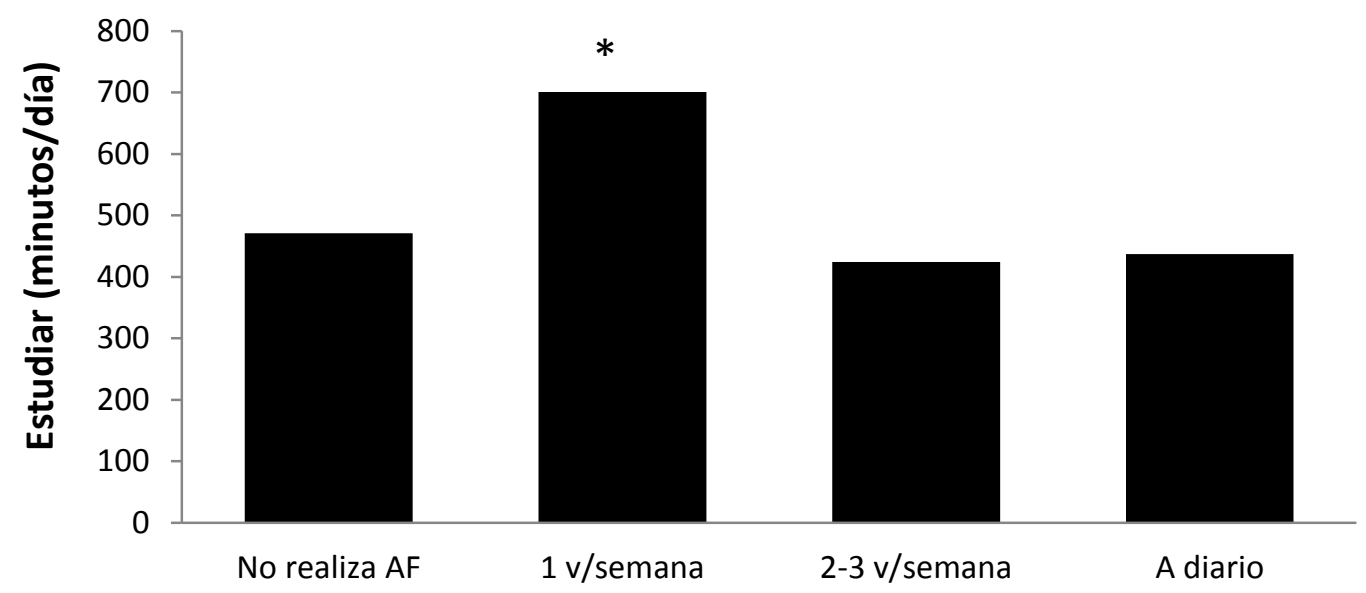

Frecuencia de ejercicio físico

Figura 1. Tiempo invertido en estudiar (min/día), según frecuencia de ejercicio físico (v/semana). Aquellos estudiantes que se ejercitan una vez a la semana dedican significativamente más tiempo al estudio. * $p<$ 0.05 estadísticamente diferente de los demás. Fuente: elaboración propia.

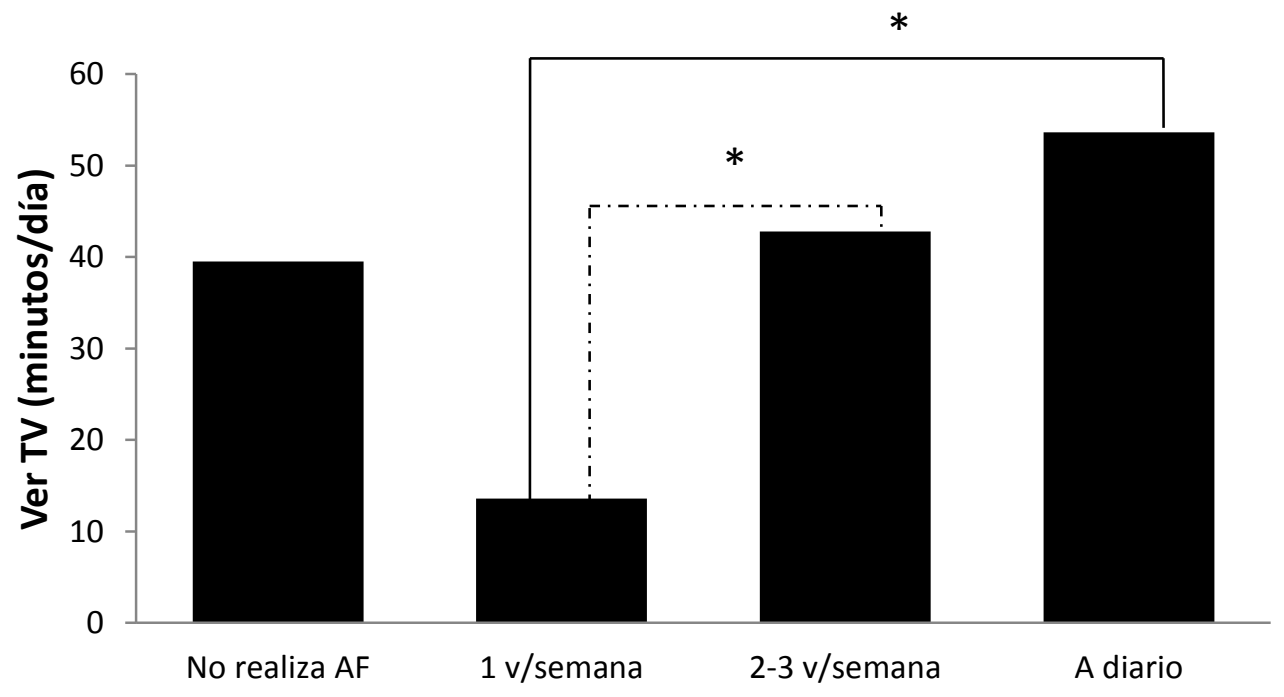

Frecuencia de ejercicio físico

Figura 2. Tiempo invertido en ver la televisión (min/día), según frecuencia de ejercicio físico (v/semana). Aquellos estudiantes que se ejercitan una vez a la semana invierten significativamente menos tiempo en ver la televisión. * $p<0.05$. Fuente: elaboración propia. 


\section{DISCUSIÓN}

Oyebode, Gordon-Dseagu, Walker y Mindell (2014) hallaron una reducción del $40 \%$ de riesgo de muerte por cualquier causa natural en quienes consumieron siete o más porciones de frutas y vegetales por día, en comparación con quienes lo hicieron menos de una vez diaria, lo cual resalta la importancia de un adecuado consumo de estos grupos de alimentos en la población estudiada en esta investigación, por ser quienes, en sus futuras vidas laborales, cuidarán la salud de muchísimas personas.

El Ministerio de Salud Pública de Costa Rica recomienda un consumo diario de 3 a 5 porciones, tanto de frutas como de vegetales (Ministerio de Salud de Costa Rica, 2011). De manera similar, y según lo planteado por Hernández-Elizondo y Sánchez-Méndez (en prensa), y Dapcich, Salvador, Pérez, Aranceta, Serra (2004), deberían ser consumidas 14 y 21 porciones semanales de vegetales y frutas, respectivamente, así como 8 porciones semanales de verduras harinosas y 7 de legumbres. Según lo detallado en la tabla 2, las y los estudiantes de medicina de la Universidad de Costa Rica consumieron, en forma estadísticamente significativa, más frutas y legumbres que lo que indican las referencias costarricenses. En cuanto a vegetales y verduras harinosas, si bien cumplieron con las recomendaciones el consumo no fue significativamente mayor a los valores de referencia.

Más de la mitad de la población estudiada (52.91\%) afirmó ingerir bebidas alcohólicas, prevalencia mayor a la reportada por Gutiérrez-Salmeán y colaboradores (2013), quienes hallaron que un $44.4 \%$ de estudiantes de medicina mexicanos consume alcohol. Dada la alta prevalencia encontrada en el presente estudio, y al tomar en cuenta que más del $20 \%$ de los sujetos inició el consumo de bebidas alcohólicas al ingresar a la universidad, sería recomendable que futuras investigaciones profundicen en la ingesta anual de alcohol puro por cada estudiante y la prevalencia de episodios de consumo masivo (binge drinking) en este sector de la población universitaria.

Por otra parte, se encontró una prevalencia de tabaquistas activos del $3.39 \%$, en forma congruente con los hallazgos de Vanderhoek y colaboradores en el año 2013 para estudiantes de medicina de la Universidad de Alberta, Canadá (3.30\%). Asimismo, los participantes del estudio presentaron un menor consumo de cigarrillos en comparación con estudiantes de medicina de Colombia (Duperly et al., 2009) y estudiantes de ciencias de la salud de México (Gutiérrez-Salmeán et al., 2013), donde las prevalencias alcanzaron el $20 \%$ y $30 \%$, respectivamente. Por lo tanto, la proporción fue menor al valor reportado para Costa Rica por la WHO en sus más recientes Estadísticas Sanitarias Mundiales (2014), las cuales indican una prevalencia en la población general de $24 \%$ en hombres y $8 \%$ en mujeres. Lo ideal sería que entre estudiantes de medicina de la Universidad de Costa Rica la prevalencia de tabaquismo fuera de $0 \%$, debido a que múltiples investigaciones han asociado el consumo de tabaco con diferentes enfermedades. Adicionalmente, Duaso et al. (2014) reportaron en su metaanálisis que los médicos tabaquistas aconsejan menos y brindan menos tratamientos a sus pacientes para cesar el hábito, en comparación con los no tabaquistas y extabaquistas.

En relación con el ejercicio, la tabla 3 muestra que un alto porcentaje de los participantes $(68.93 \%)$ dedicaba en promedio tan solo 7.78 minutos diarios a ejercitarse, cantidad que dista mucho de las recomendaciones mundiales mínimas. El $31.07 \%$ de los sujetos cumplió con las recomendaciones de la WHO (2010) y el Garber et al. (2011) en cuanto a tiempo, pero no en 
cuanto a la intensidad del ejercicio, ya que el $66.50 \%$ únicamente caminaba como forma de ejercitarse, principalmente a paso lento. Además, tanto la WHO como Garber et al. recomiendan a los adultos realizar como mínimo de 30 a 60 minutos diarios (o 150 minutos semanales) de ejercicio con intensidad moderada o de 20 a 60 minutos (o 75 minutos semanales) de ejercicio vigoroso. Estos resultados contrastan con los hallazgos de Stanford et al. (2012) y Duperly et al. (2009), quienes reportaron que un $84 \%$ y más del $50 \%$ de los estudiantes de medicina, en orden respectivo, cumplieron con las recomendaciones internacionales para actividad física. Por el contrario, los hallazgos del presente estudio muestran similitud con el comportamiento de estudiantes de México, donde más del $50 \%$ realiza muy poco o nada de ejercicio, y Perú (42.8 \% no realiza ejercicio), acorde con los hallazgos de Gutiérrez-Salmeán et al. (2013) y Bolaños y Zegarra (2010), respectivamente.

Finalmente, el análisis del tiempo destinado a actividades cotidianas, según la frecuencia semanal de actividad física, mostró que aquellas y aquellos estudiantes que realizaron ejercicio físico una vez a la semana fueron quienes, en forma estadísticamente significativa $(p<0.05)$, invirtieron más tiempo en estudiar (figura 1) y menos en ver la televisión (figura 2). Este comportamiento podría ser analizado en mayor profundidad en futuras investigaciones, para comprender mejor cómo organizan su tiempo estos estudiantes, en relación con la práctica del ejercicio.

Como futuros médicos, las y los estudiantes de medicina de la Universidad de Costa Rica tendrán un rol preponderante en la prevención primaria de enfermedades asociadas a los hábitos de alimentación, tabaquismo, ingesta de alcohol y práctica de ejercicio. Sus propios estilos de vida influirán en sus labores profesionales y en el alcance que tengan las recomendaciones que brinden a sus pacientes para motivarlos a realizar cambios positivos (Duperly et al., 2009; Shai et al., 2012; Fie, Norman \& While, 2012; Lobelo \& de Quevedo, 2014). Idealmente, debería existir un trabajo conjunto entre profesionales de medicina y profesionales del movimiento humano.

Con base en todo lo anterior, sería importante que ulteriores investigaciones determinen si el nivel de conocimiento en hábitos de alimentación saludable y ejercicio físico que reciben estos estudiantes durante su formación universitaria es suficiente e idónea, tanto para su autocuidado (Australian Medical Association [AMA], 2011) como para la orientación y recomendaciones que en el futuro brindarán a sus pacientes, pues en otros contextos se ha identificado que la formación académica en ese sentido es insuficiente (Bolaños \& Zegarra, 2010; Gutiérrez-Salmeán et al., 2013) y se ha recomendado una mejor preparación de las y los estudiantes de medicina en relación con la prescripción del ejercicio ( $\underline{\text { Holtz, Kokotilo, Fitzgerald }}$ \& Frank, 2013).

\section{CONCLUSIONES}

Existe una alta prevalencia de ingesta de bebidas alcohólicas en estudiantes de medicina de la Universidad de Costa Rica (52.91\%). En cambio, la prevalencia de tabaquismo es baja (3.39\%). El consumo diario de frutas y vegetales es estadísticamente mayor a las recomendaciones nacionales e internacionales $(p=0.001$ y $p=0.014$, respectivamente), 
mientras que el consumo diario de legumbres y verduras harinosas se ajusta a los requerimientos mínimos. A pesar de lo anterior, un alto porcentaje (40\%) de los sujetos no realizaba ejercicio físico diariamente, y entre quienes sí lo hacían el $68.93 \%$ dedicaba en promedio solamente 7.78 minutos diarios a dicha actividad. Por dicho motivo, es urgente implementar estrategias de promoción del ejercicio en esta población universitaria, para prevenir que estos futuros profesionales desarrollen sobrepeso, obesidad y enfermedades crónicas asociadas a la baja actividad física.

Además de las limitaciones propias de todo estudio transversal, esta investigación no incluyó otros grupos de alimentos como carnes, lácteos, azúcares y grasas, ni micronutrientes. En ese sentido, futuras investigaciones podrían profundizar ese apartado con un análisis nutricional completo en esta población universitaria. Por otra parte, tampoco fue posible incluir a las y los estudiantes de último año de carrera, por el tiempo sumamente limitado del cual disponen en dicha etapa de su formación, así como su dispersión geográfica en múltiples centros hospitalarios y clínicas del país. Finalmente, por limitaciones de tiempo y recurso humano no fue posible realizar mediciones antropométricas a cada participante de este estudio, ni una cuantificación detallada del comportamiento sedentario de los sujetos.

Tanto para esta población universitaria, como en futuros estudios de prevalencia con un enfoque y áreas de interés similares a los de esta investigación, sería recomendable profundizar en el análisis del comportamiento sedentario de los sujetos, y cuantificarlo (por ejemplo, a través del uso de acelerómetros) con el fin de realizar análisis más detallados en ese apartado. Asimismo, indagar en mayor profundidad los patrones de consumo de bebidas alcohólicas (tipos de alcohol, volumen de cada ingesta, frecuencia).

\section{REFERENCIAS}

Australian Medical Association. (2011). AMA position statement. Health and wellbeing of doctors and medical students. Recuperado de https://ama.com.au/position-statement/health-andwellbeing-doctors-and-medical-students-2011

Bolaños, J. J., \& Zegarra, J. W. (2010). Los futuros médicos y la actividad física: una contradicción no saludable. Apunts Med Esport, 45(167), 151-159. http://dx.doi.org/10.1016/j.apunts.2010.05.001

Caspersen, C. J., Powell, K. E., \& Christenson, G. M. (1985). Physical activity, exercise, and physical fitness: definitions and distinctions for health-related research. Public Health Reports, 100(2), 126-3. Recuperado de http://www.ncbi.nlm.nih.gov/pmc/articles/PMC1424733/

Chakravarthy, M. V., Joyner, M. J., \& Booth, F. W. (2002). An obligation for primary care physicians to prescribe physical activity to sedentary patients to reduce the risk of chronic health conditions. Mayo Clinic Proceedings, 77(2), 165-173. http://dx.doi.org/10.4065/77.2.165 
Dapcich, V., Salvador, G., Pérez, C., Aranceta, J., Serra, L. (2004). Guía de la Alimentación Saludable. Recuperado del sitio de la Sociedad Española de Nutrición Comunitaria http://aesan.msssi.gob.es/AESAN/docs/docs/publicaciones estudios/nutricion/guia alim entacion.pdf

Duaso, M. J., McDermott, M. S., Mujika, A., Purssell, E., \& While, A. (2014). Do doctors' smoking habits influence their smoking cessation practices? A systematic review and meta-analysis. Addiction, 109(11), 1811-1823. http://dx.doi.org/10.1111/add.12680

Duperly, J., Lobelo, F., Segura, C., Sarmiento, F., Herrera, D., Sarmiento, O., y Frank, E. (2009). The association between Colombian medical students' healthy personal habits and a positive attitude toward preventive counseling: cross-sectional analyses. BMC Public Health, 9, 218. http://dx.doi.org/10.1186/1471-2458-9-218

Fie, S., Norman, I. J., \& While, A. E. (2012). The relationship between physicians' and nurses' personal physical activity habits and their health-promotion practice: a systematic review. Health Education Journal, 72(1), 102-119. http://dx.doi.org/10.1177/0017896911430763

Garber, C. E., Blissmer, B., Deschenes, M. R., Franklin, B. A., Lamonte, M. J., Lee, I-M. ... Swain, D. P. (2011). Quantity and quality of exercise for developing and maintaining cardiorespiratory, musculoskeletal, and neuromotor fitness in apparently healthy adults: guidance for prescribing exercise. Medicine \& Science in Sports \& Exercise, 43(7), 13341359. http://dx.doi.org/10.1249/MSS.0b013e318213fefb

Gutiérrez-Salmeán, G., Meaney, A., Ocharán, M. E., Araujo, J. M., Ramírez-Sánchez, I., Olivares-Corichi, I. M. ... Ceballos, G. (2013). Anthropometric traits, blood pressure, and dietary and physical exercise habits in health sciences students; The Obesity Observatory Project. Nutrición Hospitalaria, 28(1), 194-201. Recuperado de http://www.redalyc.org/articulo.oa?id=309226055027

Hernández-Elizondo, J. (2009). Exposición a fitoestrógenos y su relación con la actividad física y dieta mediterránea (Tesis doctoral, Universidad de Granada España). Recuperado de http://hera.ugr.es/tesisugr/18196469.pdf

Hernández-Elizondo, J., Mariscal-Arcas, M., Rivas, A., Feriche, B., Velasco, J., \& Olea-Serrano, F. (2009). Estimación de la ingesta de fitoestrógenos en población femenina. Nutrición Hospitalaria, 24(4), 445-451. Recuperado de http://www.redalyc.org/articulo.oa?id=309226747008

Hernández-Elizondo, J. \& Sánchez-Méndez, M. (en prensa). Efecto de una intervención en familia sobre hábitos nutricionales de madres costarricenses. Actualidades Investigativas en Educación. Recuperado de http://revista.inie.ucr.ac.cr/index.php/aie

Holtz, K. A., Kokotilo, K. J., Fitzgerald, B. E., \& Frank, E. (2013). Exercise behaviour and attitudes among fourth-year medical students at the University of British Columbia. Can 
Fam Physician, 59(1), 26-32. Recuperado de http://www.ncbi.nlm.nih.gov/pubmed/23341676

Jensen, M. D., Ryan, D. H., Apovian, C. M., Ard, J. D., Comuzzie, A. G., Donato, K. A., ... Yanovski, S. Z. (2014). 2013 AHA/ACC/TOS Guideline for the management of overweight and obesity in adults: a report of the American College of Cardiology / American Heart Association Task Force on Practice Guidelines and The Obesity Society. Circulation, 129, 102-138. http://dx.doi.org/10.1161/01.cir.0000437739.71477.ee

Lobelo, F., \& de Quevedo, I. G. (2014). The evidence in support of physicians and health care providers as physical activity role models. American Journal of Lifestyle Medicine, 20(10), 1-17. http://dx.doi.org/10.1177/1559827613520120

Mariscal-Arcas, M., Romaguera, D., Rivas, A., Feriche, B., Pons, A., Tur, J. A., \& Olea-Serrano, F. (2007). Diet quality of young people in southern Spain evaluated by a Mediterranean adaptation of the Diet Quality Index-International (DQI-I). British Journal of Nutrition, 98(6), 1267-1273. http://dx.doi.org/10.1017/S0007114507781424

Ministerio de Salud de Costa Rica. (2011). Guías alimentarias para Costa Rica (2da edición). Recuperado de: http://www.ministeriodesalud.go.cr/gestores en salud/guiasalimentarias/guia alimentari as 2011 completo.pdf

Ng, M., Fleming, T., Robinson, M., Thomson, B., Graetz, N., Margono, C. ... Gakidou, E. (2014). Global, regional, and national prevalence of overweight and obesity in children and adults during 1980-2013: a systematic analysis for the Global Burden of Disease Study 2013. The Lancet, 384(9945), 766-781. http://dx.doi.org/10.1016/S0140-6736(14)60460$\underline{8}$

Organización Panamericana de la Salud. (2011). Las enfermedades no transmisibles en la región de las Américas: todos los sectores de la sociedad pueden ayudar a resolver el problema (Informe temático sobre enfermedades no transmisibles). Recuperado del http://www.paho.org/hq/index.php?option=com docman\&task=doc download\&gid=1616 0\&ltemid $=270 \&$ lang $=$ en

Oyebode, O., Gordon-Dseagu, V., Walker, A. y Mindell, J. S. (2014). Fruit and vegetable consumption and all-cause, cancer and CVD mortality: analysis of Health Survey for England data. Journal of Epidemiology \& Community Health, 68, 856-862. http://dx.doi.org/10.1136/jech-2013-203500

Shai, I., Erlich, D., Cohen, A. D., Urbach, M., Yosef, N., Levy, O., y Shahar, D. R. (2012). The effect of personal lifestyle intervention among health care providers on their patients and clinics: the Promoting Health by Self Experience (PHASE) randomized controlled intervention trial. Preventive Medicine, 55(4), 285-291. http://dx.doi.org/10.1016/i.ypmed.2012.08.001 
Stanford, F. C., Durkin, M. W., Blair, S. N., Powell, C. K., Poston, M. B. y Stallworth, J. R. (abril, 2012). Determining levels of physical activity in attending physicians, resident and fellow physicians and medical students in the USA. British Journal of Sports Medicine, 46(5), 360-364. Recuperado de http://bjsm.bmi.com/content/early/2011/12/22/bjsports-2011090299.abstract

Vanderhoek, A. J., Hammal, F., Chappell, A., Wild, T. C., Raupach, T., y Finegan, B. A. (2013). Future physicians and tobacco: an online survey of the habits, beliefs and knowledge base of medical students at a Canadian University. Tobaco Induced Diseases, 11, 1-9. http://dx.doi.org/10.1186/1617-9625-11-9

World Health Organization. (2010). Global recommendations on physical activity for health. Recuperado de http://whqlibdoc.who.int/publications/2010/9789241599979 eng.pdf?ua=1

World Health Organization. (2014). World health statistics 2014. Recuperado de http://apps.who.int/iris/bitstream/10665/112738/1/9789240692671 eng.pdf

Participación: A- Financiamiento, B- Diseño del estudio, C- Recolección de datos, D- Análisis estadístico e interpretación de resultados, E- Preparación del manuscrito. 